\title{
Our Success in the Treatment of Acute Respiratory Failure in Coronovirus Patients Using Non-invasive Ventilation
}

\author{
Polukhov RSh, Nasibova EM*, Polukhova AE, Rustamkhanly AM, Sultanova MJ and Nasibov FG \\ Azerbaijan Medical University, Baku
}

*Corresponding author: Nasibova EM, Azerbaijan Medical University, Baku

\begin{abstract}
The novel coronavirus infection (COVID-19) pandemic, caused by the highly contagious severe acute respiratory syndrome coronavirus (SARS-CoV-2), is still at its peak, causing thousands of deaths every week. Since the beginning of the coronavirus (just a few months ago), our understanding of the disease has grown rapidly. While there are several large, randomized drug trials currently underway, current survival from severe COVID-19 is entirely dependent on providing the best supportive care possible.
\end{abstract}

Keywords: Non-invasive ventilation, Acute respiratory failure, COVID-19

\section{Introduction}

For patients with acute respiratory failure with suspected COVID-19 or with confirmed COVID-19, the strategy and tactics of respiratory therapy and respiratory support do not differ significantly from those for parenchymal acute respiratory failure of any genesis (community-acquired bacterial or viral pneumonia, nosocomial, including respirator-associated pneumonia, ARDS) $[1,2]$. The strategy and tactics of choosing methods of respiratory support and correction of parameters of mechanical ventilation in severe acute respiratory failure caused by a new viral infection COVID-19 is, in principle, similar to those in ARDS caused by direct damaging factors [3-6]. The basic principles of the effectiveness of treatment of severe parenchymal acute respiratory failure of various origins are as follows:
a. timely differential diagnosis of acute respiratory failure.
b. timely identification and adequate correction of risk factors and predisposing factors of lung damage in intensive care units.
c. adequate treatment of the leading causes of acute respiratory failure.
d. rational use of non-invasive artificial ventilation.

e. implementation of the principles of sparing artificial ventilation.

f. differentiated use of respiratory strategies.

g. differentiated use of adjuvant methods for correcting hypoxia.

h. complex treatment of patients with acute respiratory failure - treatment of acute respiratory failure, this treatment is not only the lungs.

i. To clarify the nature and extent of lung damage for all patients with ARDS, suspected COVID-19 or confirmed COVID-19, it is advisable to perform computed tomography of the lungs, provided this method is available and the patient is transportable. In cases of viral pneumonia, when performing frontal radiography of the lungs, there is a significantly lower efficiency of detecting changes than with CT. With ARDS and viral pneumonia, CT allows you to:

j. to identify pathological changes in the lungs (bilateral polysegmental infiltrates) in the early stages of the disease even before the development of signs of acute respiratory failure. 
k. assess the dominant cause of acute respiratory failure (diffuse atelectasis, overhydration, fibrosis / hyalinosis, etc.).

l. assess lung recruitment.

m. stratify patients in relation to the decision of questions about hospitalization, the choice of treatment tactics and methods of respiratory support $[1,2,4,5,7,8]$.

Considering that acute respiratory failure in ARDS and viral pneumonia has similar fundamental mechanisms of pathogenesis (decrease in thoracopulmonary compliance, decrease in functional residual capacity, accumulation of extravascular fluid in the lungs, deterioration of regional ventilation-perfusion relations and an increase in intrapulmonary shunting of blood), in the case of hypoxemia it appears it is inappropriate to carry out traditional oxygen therapy (through nasal cannulas or a face mask) [9]. Indeed, the use of a low-flow (4 - $8 \mathrm{l} / \mathrm{min}$ ) air-oxygen mixture will not allow to effectively influence the basic mechanisms of the pathogenesis of acute respiratory failure and increase arterial blood oxygenation with a high risk of damage to the epithelium of the upper respiratory tract and alveoli with poorly moistened and poorly warmed oxygen [10]. Therefore, with the development of parenchymal acute respiratory failure and with mild ARDS of any etiology, it is advisable to use non-invasive respiratory support (NIV) methods. The development of modern medical technologies has made it possible to widely introduce various NIV methods into clinical practice. It is used at different stages of the treatment of respiratory failure of various origins: to reduce the frequency of intubation, for treatment as an alternative to invasive ventilation, at the stage of stopping ventilation (the possibility of earlier extubating), after extubating (reducing the frequency of reintubation and resumption of mechanical ventilation) $[10,11]$. Non-invasive respiratory support improves lung biomechanics and gas exchange and reduces the patient's work of breathing. These effects are realized through the application of positive end expiratory pressure (PEEP) or continuous positive airway pressure (CPAP) and inspiratory pressure support (PS). Positive effects of PEEP (CPAP): an increase in the functional residual lung capacity and their elasticity, a decrease in regional ventilationperfusion disorders, an improvement in gas exchange in the lungs, a decrease in the patient's respiratory work, a decrease in transpulmonary pressure, a decrease in the negative respiratorycirculatory effects of NIV and / or mechanical ventilation. Modern respiratory technologies offer a wide range of NIV modes: CPAP, EPAP, PSV, PPS, VS, VTBS, BIPAP, DuoPAP, ICPAP, ASV, etc. There is a sufficient selection of devices for connecting the respirator to the patient's airway: nasal cannulas, tracheostomy cannulas, nasal mask, face mask, Total face, helmet, etc. [2,12]. In patients with parenchymal acute respiratory failure, ARDS, pneumonia, NIV is the starting method of respiratory support, especially in indirect ARDS. The main indications for NIV in this contingent are: dyspnea at rest, respiratory rate $(\mathrm{RR})>25-30$ breath/min; participation in respiration of auxiliary muscles, thoracoabdominal asynchronism, hypoxemia $-\mathrm{PaO}_{2} \leq 60, \mathrm{SaO}_{2} \leq 90 \%$ (with $\mathrm{FiO}_{2}=0.4-0.5$ or $\mathrm{PaO}_{2} / \mathrm{FiO}_{2}$ $<300$ with $\mathrm{FiO}_{2}=0.21$, Qs / Qt $\geq 10 \%$ ); hypercapnia $-\mathrm{PaCO}_{2}>45 \mathrm{~mm}$ $\mathrm{Hg}$ Art. and / or its progressive increase; hypocapnia $-\mathrm{PaCO}_{2}<7.35$ and / or its progressive decrease; increased airway resistance (Raw) is 1.5-2.0 times higher than normal; tachycardia at rest (heart rate> 90 per minute); arterial hypertension (hypotension), acrocyanosis, cyanosis, a combination of the above factors [10,7,11]. Modern respiratory technologies make it possible to implement various modes of respiratory support in NIV conditions with a wide range of settings for ventilation parameters in patients of different profiles.

In several clinical situations, NIV is an effective alternative to mechanical ventilation. The advantages of NIV over mechanical ventilation:

1. absence of complications inherent in tracheal intubation and long-term presence of an endotracheal tube in it.

2. lower risks of developing respiratory complications.

3. lower risks of developing circulatory complications.

4. reducing the risks of developing nosocomial infections.

5. reducing the need for medication sedation.

6. the possibility of contact with the patient (the possibility of earlier mobilization of the patient).

7. pharmacoeconomic benefits. It should be remembered that there are many advantages of maintaining spontaneous breathing with respiratory support in patients with ARF of various origins: prevention of atrophy of the respiratory muscles; improvement of regional ventilation-perfusion relations; improving cardio hemodynamics, reducing the load on the right ventricle of the heart; maintaining the cough reflex - reducing the risk of developing respirator-associated pneumonia; facilitating the process of weaning from mechanical ventilation; reduction in the duration of respiratory support and stay in the intensive care unit $[6,11,13]$. However, any NIV method is not devoid of negative effects.

The main disadvantages of NIV:

1) the need for active cooperation of medical personnel with the patient.

2) the complexity of setting the NIV parameters.

3) the need for frequent correction of NIV parameter settings.

4) impossibility to realize high PEEP under NIV conditions.

5) impossibility to realize high inspiratory pressure under NIV conditions.

6) lack of direct access to the respiratory tract for sanitation 
in NIV conditions.

7) high risk of aerophagia.

8) high risk of aspiration of the contents of the oral cavity and stomach.

9) maceration and necrosis of the skin at the points of contact of the mask.

10) nosebleeds.

11) conjunctivitis.

12) the possibility of developing hypoxemia with a leaky mask connection.

13) inadequate heating and humidification (conditioning) of the gas mixture.

14) risks of equipment contamination, infection of medical personnel and patients with highly contagious infections $[3,6,12]$.

Despite all the obvious advantages of NIV, in several clinical situations this method cannot replace mechanical ventilation.

Absolute contraindications for any NIV methods are:

a. lack of spontaneous breathing.

b. lack of consciousness.

c. $\mathrm{PaO}_{2} / \mathrm{FiO}_{2} \leq 170$.

d. impossibility of implementing NIV (craniofacial trauma, damage to the upper respiratory tract, constitutional features).

e. lack of cooperation with the patient. Given the specifics of NIV, its effectiveness may be significantly reduced, or the implementation of this method becomes impossible. This can be in the following clinical situations:

f. inability to protect the respiratory tract (impaired coughing and swallowing) and a high risk of aspiration.

g. excessive bronchial secretion.

h. impairment of consciousness (agitation or depression of consciousness).

i. severe obesity.

j. active bleeding from the gastrointestinal tract.

k. obstruction of the upper airways.

l. discomfort from the mask, claustrophobia.

m. operations on the upper respiratory tract.

n. the patient's serious condition or its progressive deterioration (unstable hemodynamics, myocardial ischemia, life-threatening arrhythmias, severe metabolic disorders and/ or dyshidria, uncontrolled arterial hypertension) [3,6,12].

\section{Objective}

To determine the benefits of NIV in ARF in patients with COVID-19.

\section{Research Material}

The study included 25 patients admitted to the intensive care unit of the AMU surgical clinic during the period from April 1 to August 1, 2020.

\section{Research Results}

Our experience with NIV has shown that most patients undergoing NIV tolerate this procedure relatively well at the initial stage. However, in a number of patients during the first minutes or hours of NIV, there is no improvement in their condition (clinical parameters and gas exchange) or the procedure is poorly tolerated; the proportion of such patients is usually about 15-35\%. During the period of work with patients with COVID-19, we have developed an algorithm for selecting the primary parameters of NIV:

a. choose the starting level of PEEP 4-6 cm of water. Art.

b. evaluate the patient's DO, signs of increased work of breathing (shortness of breath, participation of auxiliary muscles, thoracoabdominal synchronism, etc.).

c. set the minimum trigger sensitivity at which there is no auto trigging (-1.5-2.0 $\mathrm{cm} \mathrm{H}_{2} \mathrm{O}$ for a pressure trigger: $2-3 \mathrm{l} / \mathrm{min}$ for a flow trigger).

d. select the level of inspiratory pressure support (PS, IPAP) individually by means of a stepwise increase (1-2 cm of water column) from 4-6 cm of water. Art. until a tidal volume equal to $6-8 \mathrm{ml} / \mathrm{kg}$ of the proper body weight (DMT) is reached (calculation of DMT $(\mathrm{kg}):$ men $=50+0.91 \times$ (height, $(\mathrm{cm})-$ 152.4); women $=45.5+0.91 \times($ height, $(\mathrm{cm})-152.4)$, estimate $\mathrm{SpO}_{2}, \mathrm{RR}, \mathrm{DO} / \mathrm{RR}$;

e. adjust the amount of acceleration of the peak inspiratory flow adequate to the patient's current state (corresponding to the state of his neuro-respiratory drive - "respiratory demand" - in accordance with the patient's inspiratory rate), it is advisable to focus on the graphic monitoring of respiration - to avoid "flow hunger" and excessively high rate of rise flow;

f. configure the trigger for switching the device from inhalation to exhalation to ensure sufficient DO and patient comfort (it is advisable to use the algorithm for selecting a percentage decrease in the flow rate from its maximum value), you should focus on the graphical monitoring of respiration -to achieve compliance with the duration of the apparatus inspiration with the duration of the patient's inspiration; 
g. set the inspiratory fraction of oxygen in the inhaled gas mixture $\left(\mathrm{FiO}_{2}\right)$ at the minimum level that ensures $\mathrm{SpO}_{2} 88$ $95 \%$.

h. in patients with $\mathrm{SpO}_{2}<88 \%$ against a background of $\mathrm{FiO}_{2}$ $=0.3$, gradually $(1-2 \mathrm{~cm}$ of water column) increase PEEP to 8-12 cm of water. Art. subject to portability.

i. use respiratory and cardio monitoring.

j. use graphical monitoring of NIV.

k. focus on the subjective state of the patient.

l. constantly adjust the respirator settings.

It should be especially noted that the adjustment of NIV parameters should be started with a gradual increase in PEEP. In many cases, optimal PEEP can quickly correct respiratory distress. Pressure support is advisable in the presence of thoracoabdominal asynchronism and/or tidal volume/respiratory rate $>105$. Inadequately selected inspiratory and expiratory triggers accelerated peak inspiratory flow, and inspiratory/expiratory ratios can significantly reduce the therapeutic efficacy of NIV. In most cases, this algorithm for selecting the starting NIV settings ensures its effectiveness and safety. It should be remembered that the wrong choice of any of these NIV parameters will not allow to realize all the therapeutic possibilities of this method of respiratory support, reduce its clinical effectiveness and lead to the need for tracheal intubation and initiation of invasive controlled ventilation. It should be borne in mind:

i. high values of PEEP (10-12 $\mathrm{cm}$ of water column and more) and/or PS 16-18 cm of water. Art. and more), despite a temporary improvement in oxygenation, lead to patient discomfort and increase the risk of developing lung damage.

ii. dyspnea reduction is usually achieved shortly after adequate ventilation has been set, while hypercapnia and/or hypoxemia may take several hours to correct.

iii. during the first hours, the NIV should be carried out continuously.

iv. monitoring of gas exchange parameters and the patient's condition during the first hours of NIV should be carried out continuously.

v. further, after the improvement of the gas exchange indices, a gradual decrease in the NIV intensity is possible (first, a decrease in the oxygen fraction, then the PS value, then the PEEP level).

vi. further, with stable indicators of gas exchange and the patient's condition, it is possible to carry out NIV sessions for several hours a day, depending on the patient's condition and tolerance. vii. it is advisable to combine NIV sessions with sessions of high-flow oxygen therapy.

At the beginning of NIV, a fundamental condition is careful monitoring of the patient's condition and a rapid (within 15$30 \mathrm{~min}$ ) assessment of the effectiveness of respiratory support, especially in ARDS and viral pneumonia. When assessing the clinical effectiveness of NIV, a comprehensive assessment of the patient's condition is required. Pay attention:

a. to decrease $\mathrm{PaO}_{2} / \mathrm{FiO}_{2}<175$.

b. lack of growth of $\mathrm{PaO}_{2} / \mathrm{FiO}_{2}$.

c. growth of PaCO.

d. an increase in respiratory rate $>25-30$ per minute or no decrease in respiratory rate.

e. an increase in the ratio of respiratory rate/tidal volume $>100$.

f. the emergence or growth of encephalopathy.

g. desynchronization of the patient with the respirator.

h. appearance or increase of acidosis.

i. deterioration of the patient's condition.

The criterion for successful NIV in our study was the improvement of arterial blood gas composition and the ability to avoid endotracheal intubation.

\section{Clinical Case}

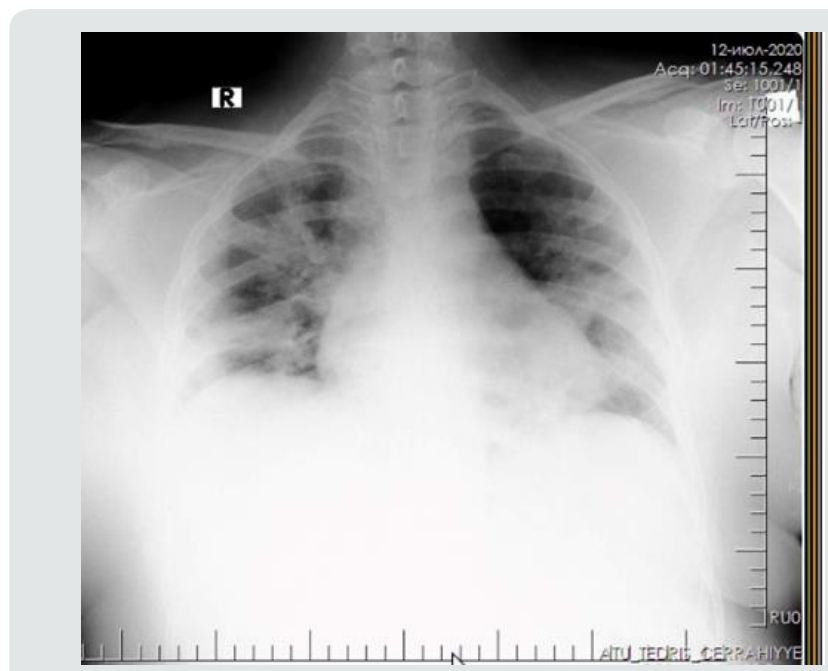

Figure 1: X-ray of the lungs on admission.

The patient is a woman aged 40 , with complaints of intermittent chronic cough, temperature $39.3^{\circ} \mathrm{C}$, chills, headache, shortness of breath, loss of appetite, loss of the sense of smell. On auscultation of the lungs, crepitus, moist rales in the lower lobes of the lungs, oxygen saturation $86-88 \%$, respiratory rate $26-28$ per minute were 
noted. Laboratory tests on admission included: WBC $-18.42 \times 109 / \mathrm{L}$, LYM - 0.51x109/L, albumin -3.11g/dL, PI - 51.9\%, PT -14.8 sec., INR 1.39, Fibrinogen $128 \mathrm{mg} / \mathrm{L}$, GRP $27.4 \mathrm{mg} / \mathrm{L}$, D-Dimer $>7500 \mathrm{ng} / \mathrm{mL}$, Ferritin $1088 \mathrm{ng} / \mathrm{mL}, \mathrm{P} / \mathrm{F}>150$. Chest X-ray and CT are indicated in the following (Figures 1-9).

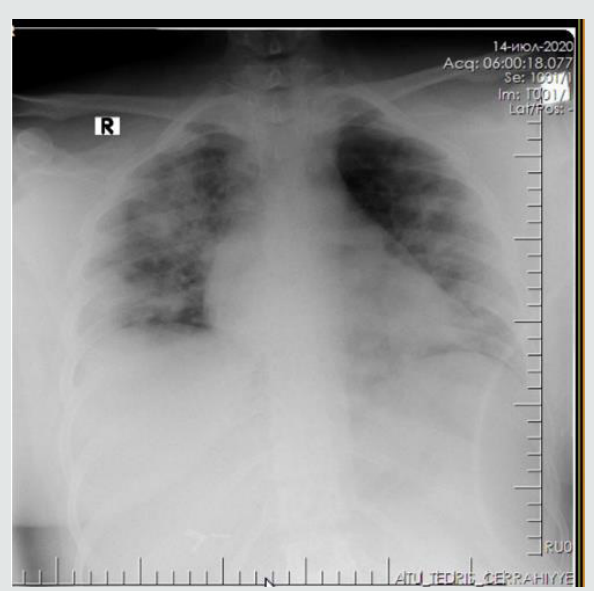

Figure 2: X-ray of the lungs on admission.

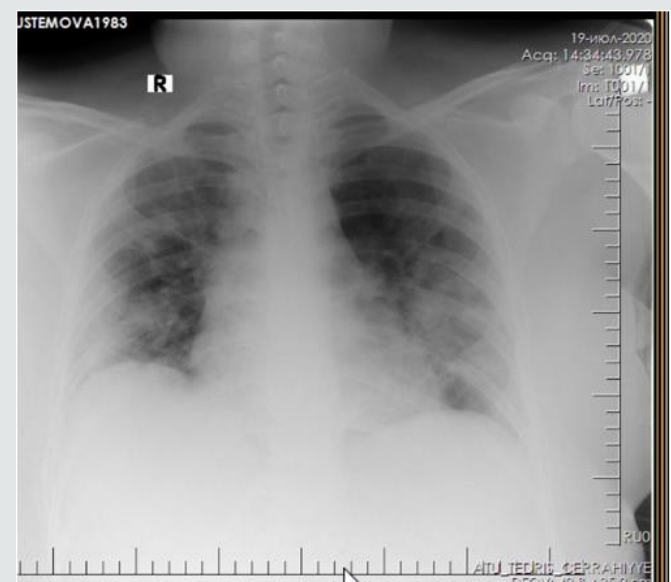

Figure 3: Radiograph of the lungs after improvement.

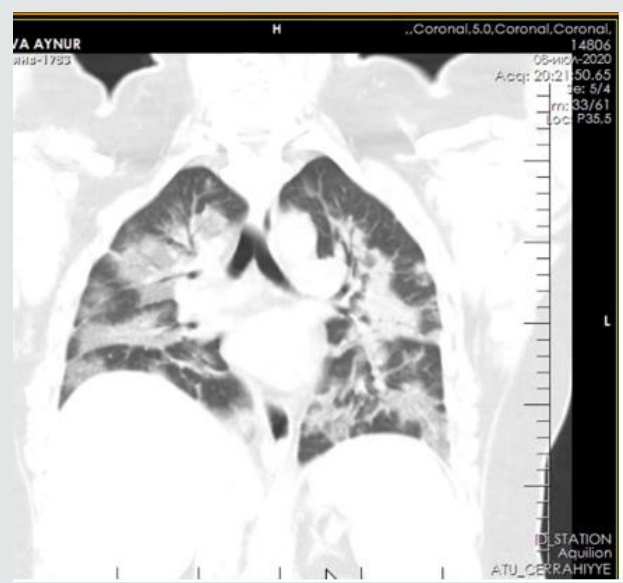

Figure 4: CT of the lungs at admission.

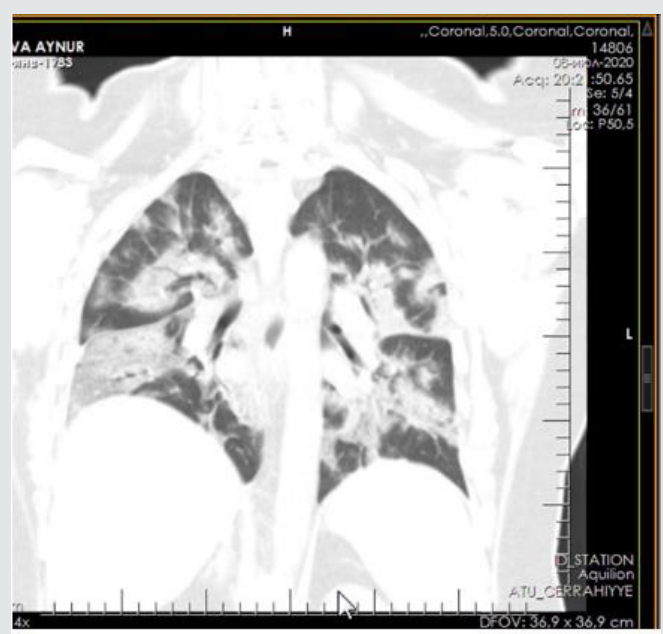

Figure 5: CT of the lungs on the $3^{\text {rd }}$ day after treatment.

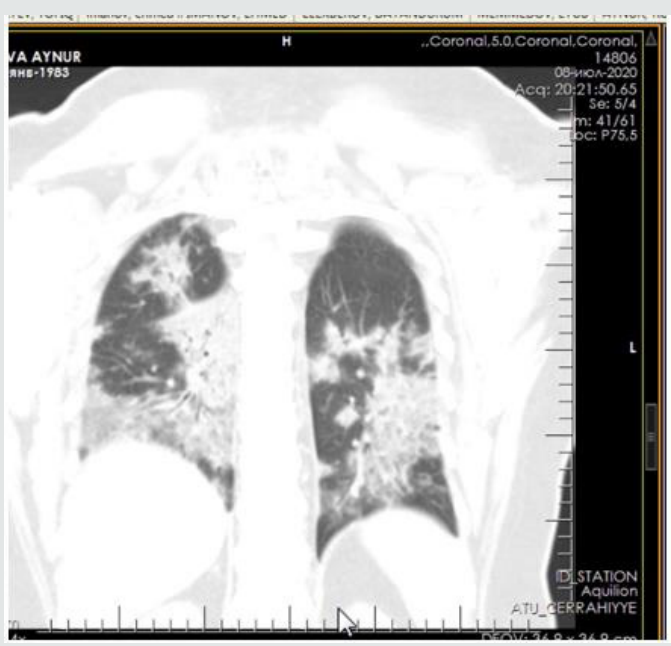

Figure 6: CT of the lungs on the $7^{\text {th }}$ day after treatment.

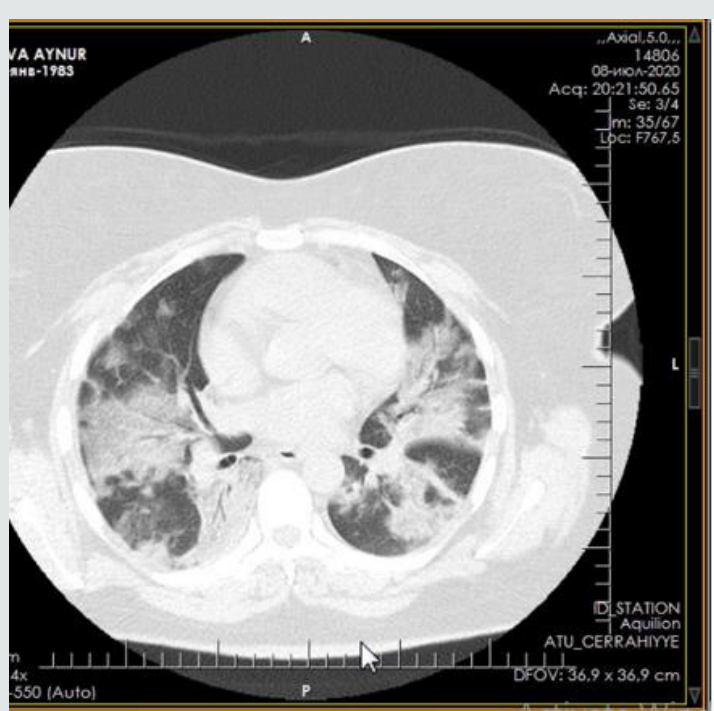

Figure 7: CT of the lungs on the $9^{\text {th }}$ day after treatment. 


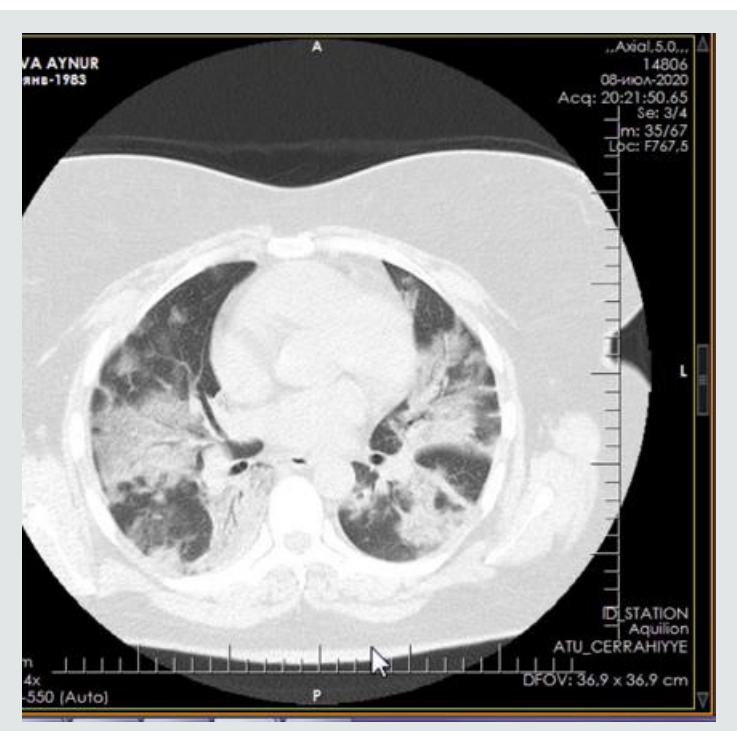

Figure 8: CT of the lungs on the $11^{\text {th }}$ day after treatment.

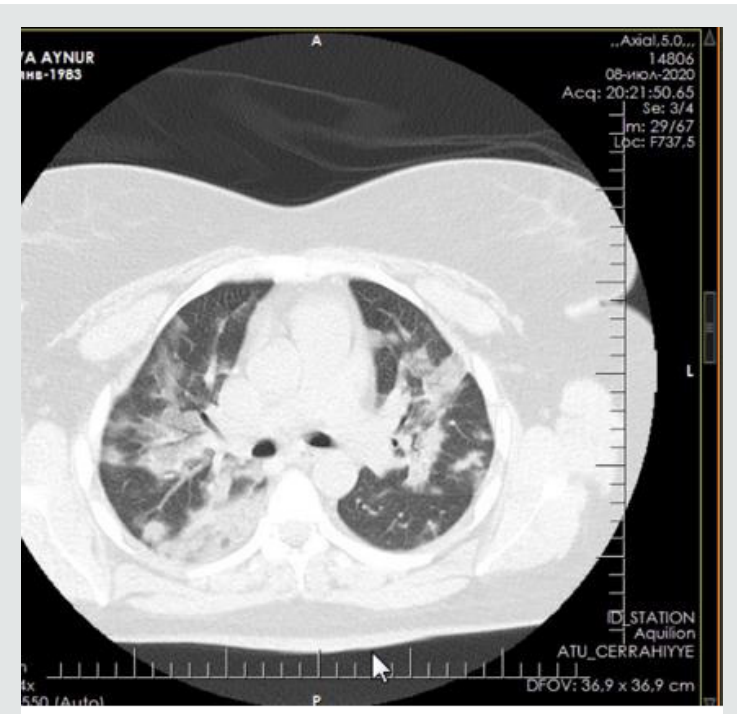

Figure 9: CT of the lungs 15 days after treatment.

Non-invasive ventilation of the lungs was carried out with an oral-nasal mask using an ELISA ventilator. The parameters were set and adjusted according to the general state and according to blood gas data 4 times a day: $\mathrm{RR}<35, \mathrm{pH}>7.30$, neurological dysfunction according to the Kelly scale $>3-5$, modified with a scale for determining the participation of auxiliary respiratory muscles $<3$ points. With hypercapnia, the following parameters were set Ps -12 , PEEP - $6 \mathrm{~cm}$ water column, $\mathrm{FiO}_{2}-30-40 \%$, and with hypoxemia -Ps12 , PEEP $-5 \mathrm{~cm}$ water column, $\mathrm{FiO}_{2}-50-60 \%$. The median treatment period with NIV was 12 days. The average daily treatment time with NIV on the first day was 16.5 hours, on the second day -17.2 hours and on the third day 15.7 hours. The patient was discharged on the $18^{\text {th }}$ day with improvement.

\section{Conclusion}

With the development of ARF of any etiology, timely differential diagnosis is important, considering all the predisposing factors for the development of respiratory disorders. Early use of NIV with correct settings of starting parameters, combined and combined use of different NIV methods, positional and respiratory therapy, respiratory simulators, etc. are fundamentally important; comprehensive monitoring and thorough assessment of the patient's condition, timely resolution of the issue of the need for intubation; comprehensive monitoring and adjuvant therapy. It has been proven that NIV avoids tracheal intubation, reduces the risk of complications, mortality and length of hospital stay.

\section{References}

1. Bein B, Bachmann M, Huggett S, Wegermann P. SARS CoV-2/COVID-19: evidence-based recommendation on diagnosis and therapy. Anasthesiol Intensivmed Notfallmed Schmerzther Europe PMC 55(4): 257-265.

2. Bellani G, John G Laffey, Tài Pham, Fabiana Madotto, et al. (2017) Noninvasive ventilation of patients with acute respiratory distress syndrome. Insights from the LUNG SAFE study. Am J Respir Crit Care Med 195: 67-77.

3. Chandra D, Stamm JA, Taylor B, Rose Mary Ramos, Lewis Satterwhite, et al. (2012) Outcomes of noninvasive ventilation for acute exacerbations of chronic obstructive pulmonary disease in the United States, 19982008. Am J Respir Crit Care Med 185(2): 152-159.

4. Rochwerg B, Laurent Brochard, Mark W Elliott, Dean Hess, Nicholas S Hill, et al. (2017) Official ERS/ATS clinical practice guidelines: noninvasive ventilation for acute respiratory failure. Eur Respir J 50: 17-25.

5. Fisher KA, Mazor KM, Goff S, Mihaela S Stefan, Penelope S Pekow, et al. (2017) Successful use of noninvasive ventilation in chronic obstructive pulmonary disease. How do high-performing hospitals do it? Ann Am Thorac Soc 14(11): 1674-1681.

6. Zareifopoulos N, Lagadinou V, Karela A, Gerasimos Karantzogiannis, Dimitrios Velissaris (2020) Intubation and mechanical ventilation of patients with COVID-19: what should we tell them?. Monaldi Arch Chest Dis 90(1).

7. Ergan B, Nasiłowski J, Winck JC (2018) How should we monitor patients with acute respiratory failure treated with noninvasive ventilation? Eur Reapiratory Rev 148: 27-35.

8. Scala R, Pisani L (2018) Noninvasive ventilation in acute respiratory failure: which recipe for success? Eur Respir Rev 27: 18-29.

9. Gattinoni L, Chiumello D, Rossi S (2020) COVID-19 pneumonia: ARDS or not? Crit Care 24(1): 154.

10. Hess DR, Pang JM, Camargo CA Jr (2009) A survey of the use of noninvasive ventilation in academic emergency departments in the United States. Respir Care 54(10): 1306-1312.

11. Yang X, Yu Y, Xu J, Huaqing Shu, Jiaan Xia, et al. Clinical course and outcomes of critically ill patients with SARS-CoV-2 pneumonia in Wuhan, China: a single-centered, retrospective, observational study. Lancet Respir Med 3079-3085.

12. Correa TD, Paula RS, Lúbia Caus de Morais, Farah Christina Scarin, Eliézer Silva, et al. (2015) Performance of noninvasive ventilation in acute respiratory failure in critically ill patients: a prospective, observational, cohort study. BMC Pulmonary Medicine 144: 15-25.

13. McLaughlin KM, Murray IM, Thain G, Currie GP (2010) Ward-based noninvasive ventilation for hypercapnic exacerbations of COPD: a 'reallife' perspective. QJM Int J Med 103(7): 505-510. 
CC (i) This work is licensed under Creative Commons Attribution 4.0 License

To Submit Your Article Click Here: Submit Article

DOI: $10.32474 /$ GJAPM.2020.03.000172

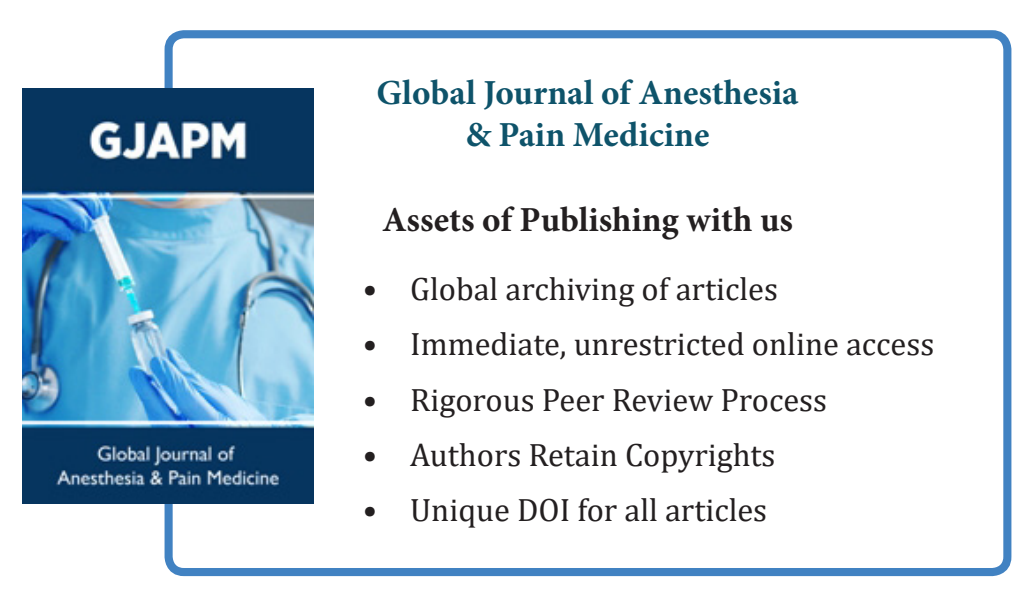

